\title{
Ibuprofen and fatal lung cancer: A brief report of the prospective results from the Third National Health and Nutrition Examination Survey (NHANES III)
}

\author{
MARISA A. BITTONI ${ }^{1,2}$, DAVID P. CARBONE ${ }^{1,2}$ and RANDALL E. HARRIS ${ }^{1,3}$ \\ ${ }^{1}$ Comprehensive Cancer Center, The Ohio State University; ${ }^{2}$ Division of Thoracic Oncology, College of Medicine; \\ ${ }^{3}$ Division of Epidemiology, College of Public Health, The Ohio State University, Columbus, OH 43210, USA
}

Received November 8, 2016; Accepted March 4, 2017

DOI: $10.3892 /$ mco.2017.1239

\begin{abstract}
Chronic inflammation appears to increase the risk of lung cancer and, reciprocally, agents that reduce inflammation have been found to reduce this risk. However, few prospective studies have assessed whether there exists an association between lung cancer and the use of non-steroidal anti-inflammatory drugs (NSAIDs). In the present study, the association between fatal lung cancer and NSAIDs was investigated using cohort data from the Third National Health and Nutrition Examination Study (NHANES III). Baseline data were collected on smoking, NSAID use and other lifestyle factors for 10,735 participants during 1988-1994, with cause-specific mortality status ascertained through probabilistic record matching based on the National Death Index until 2006. Cox proportional hazards regression models were conducted to estimate hazard ratios (HRs) and confidence intervals (CIs) for NSAID use and death from lung cancer, controlling for current smoking and other covariates. During the 18 years of follow-up, 269 participants succumbed to lung cancer, of whom $252(93.6 \%)$ reported a history of cigarette smoking. Since all but 17 of the 269 fatal lung cancer cases occurred among current or former smokers, estimates of NSAID effects were ascertained from a sub-cohort of 5,882 individuals who reported a history of past or current cigarette smoking. Multivariate regression models revealed that regular use of ibuprofen resulted in a $48 \%$ reduced risk of lung cancer mortality $(\mathrm{HR}=0.52,95 \% \mathrm{CI}$ : $0.33-0.82, \mathrm{P}<0.01)$. The main effects of other compounds tested, such as aspirin or acetaminophen, were not statistically significant. Our results suggest that high-risk subgroups of smokers may benefit from the regular use of specific NSAIDs, which may prove to be a useful strategy for lung cancer prevention.
\end{abstract}

Correspondence to: Dr Marisa A. Bittoni, Comprehensive Cancer Center, The Ohio State University, 482 Biomedical Research Tower, Columbus, $\mathrm{OH} 43210$, USA

E-mail: bittoni.1@osu.edu

Key words: ibuprofen, aspirin, acetaminophen, non-steroidal anti-inflammatory drugs

\section{Introduction}

Lung cancer is known to be the leading cause of cancer mortality worldwide in men and women, accounting for $\sim 28 \%$ of total cancer deaths. In 2012, the World Health Organization reported 1.82 million new cases of lung cancer and 1.59 million lung cancer deaths (1).

Smoking tobacco, which is the dominant risk factor associated with lung cancer development, accounts for $80-90 \%$ of deaths from lung cancer in men and women. Environmental/ occupational exposure, such as environmental tobacco smoke, asbestos and radon, along with inherited genetic susceptibility, may affect lung carcinogenesis (2).

A meta-analysis based on 18 epidemiological reports of non-steroidal anti-inflammatory drug (NSAID) use and lung cancer revealed a $28 \%$ reduction in lung cancer risk among individuals who reported regularly taking non-prescription NSAIDs, such as aspirin and ibuprofen (3). However, case-control studies and those adjusted for smoking exhibit a marginally greater risk reduction compared with cohort studies and those not adjusted for smoking (4). In the present investigation, data from the Third National Health and Nutrition Examination Survey (NHANES III) were prospectively examined to determine the association of aspirin or ibuprofen use with fatal lung cancer among cigarette smokers.

\section{Patients and methods}

Population characteristics. Study data were obtained from NHANES III, which was a complex, multi-stage, stratified sample of civilian, non-institutionalized individuals in the USA (5). The participants in NHANES III were interviewed during 1988-1994 and were followed until death or December 1, 2006. A total of 10,735 participants aged $\geq 40$ years and cancer-free at the start of the study were included in the analysis. Trained interviewers collected questionnaire data on demographic and lifestyle factors, including race/ethnicity, socioeconomic status, medical history, medication use and smoking history (6).

NSAID intake. The participants were specifically questioned regarding their intake of over the counter pain medications 
Table I. Baseline characteristics of adults aged $>40$ years comparing cases of fatal lung cancer with non-cases (NHANES III).

\begin{tabular}{|c|c|c|c|}
\hline Variables & $\begin{array}{l}\text { Lung cancer } \\
\qquad(n=269)\end{array}$ & $\begin{array}{l}\text { Non-cases } \\
(n=10,466)\end{array}$ & $\begin{array}{c}\text { Total } \\
(\mathrm{n}=10,735)\end{array}$ \\
\hline \multicolumn{4}{|l|}{ Gender } \\
\hline Male & $180(66.9)$ & $4,921(47.0)$ & $5,101(47.5)$ \\
\hline Female & $89(33.1)$ & $5,545(53.0)$ & $5,634(52.5)$ \\
\hline \multicolumn{4}{|l|}{ Race $^{\mathrm{a}}$} \\
\hline White & $177(73.0)$ & $7,633(73.0)$ & $7,810(72.8)$ \\
\hline African American & $87(32.3)$ & $2,559(24.5)$ & $2,153(24.7)$ \\
\hline Other & $5(1.9)$ & $270(2.6)$ & $241(2.6)$ \\
\hline \multicolumn{4}{|l|}{$\mathrm{BMI}^{\mathrm{b}}, \mathrm{kg} / \mathrm{m}^{2}$} \\
\hline$<18.5$ underweight & $41(15.2)$ & $1,389(13.3)$ & $1,430(13.3)$ \\
\hline $18.5-24.9$ normal & $104(38.9)$ & 2,937 (28.1) & $3,041(28.3)$ \\
\hline 25.0-29.9 overweight & $83(30.9)$ & $3,531(33.7)$ & $3,614(33.7)$ \\
\hline$>30$ obese & $41(15.2)$ & $2,609(24.9)$ & $2,650(24.7)$ \\
\hline \multicolumn{4}{|l|}{ Smoking status ${ }^{\mathrm{b}}$} \\
\hline Ever smoker ( $\geq 100$ cigarettes) & $252(93.7)$ & $5,630(53.9)$ & $5,882(54.9)$ \\
\hline Former & $108(40.2)$ & $3,400(32.5)$ & $3,508(32.7)$ \\
\hline Current & $144(53.4)$ & $2,230(21.3)$ & $2,374(22.1)$ \\
\hline \multicolumn{4}{|l|}{ Comorbidities $^{\mathrm{c}}$} \\
\hline Yes & $137(50.9)$ & $5,013(47.9)$ & $5,150(48.0)$ \\
\hline No & $132(49.1)$ & $5,453(52.1)$ & $5,585(52.0)$ \\
\hline
\end{tabular}

${ }^{\mathrm{a}} \mathrm{P}<0.05 .{ }^{\mathrm{b}} \mathrm{P}<0.0001$. ${ }^{\mathrm{c}}$ Congestive heart failure, stroke, hypertension, heart attack and diabetes. NHANES III, Third National Health and Nutrition Examination Study; BMI, body mass index.

in the last month, as well as how often each medication was taken. These consisted of the following: Aspirin (included Anacin, Bufferin, Ecotrin, Ascriptin and Midol), acetaminophen (included Tylenol and Anacin-3) and ibuprofen (included Advil, Nuprin and Medipren). We hypothesized that reported NSAID use in the previous month would be consistent with long-term regular use. The outcome measure was death due to lung cancer, which was ascertained through record linkage using the National Death Index (7).

Statistical analysis. Since $252(93.6 \%)$ of the 269 fatal lung cancer cases occurred among smokers, the effects of aspirin, ibuprofen and acetaminophen were estimated from a sub-cohort of 5,882 individuals who reported a history of current or former cigarette smoking. The effects were calculated by estimating hazard ratios (HRs) with $95 \%$ confidence intervals (95\% CIs) and associated P-values from Cox regression models. Statistical analyses were conducted using SAS 9.3 software (SAS Institute Inc., Cary, NC, USA).

\section{Results}

Cohort characteristics. The characteristics of the NHANES III cohort of 10,735 adults aged $>40$ years, among whom 269 fatal lung cancer cases were detected during 18 years of follow-up, are summarized in Table I. Approximately two-thirds of lung cancer cases were male and $94 \%$ had ever smoked. Non-cases were more likely to be overweight/obese compared with cases.
HRs were estimated for current and past smokers $(\mathrm{HR}=13.2$ and 9.8, respectively), reflecting the dominant effect of smoking on lung cancer risk.

Sub-cohort analysis. The results of regression analyses for the sub-cohort of NHANES III containing only 5,882 adults who reported a history of current/former smoking at the baseline interview are presented in Table II. Estimates of HRs assessed the lung cancer mortality risk for individuals reporting intake of aspirin, ibuprofen or acetaminophen, compared with those who reported no use of any of these compounds. Multivariate regression models revealed that regular use of ibuprofen reduced the lung cancer death risk by $48 \%$ ( HR $=0.52,95 \%$ CI: $0.33-0.82$, $\mathrm{P}<0.01)$. A significant inverse trend in this risk was observed for increased frequency of ibuprofen intake $(\mathrm{P}<0.02$, Fig. 1). The effects of aspirin and acetaminophen were not statistically significant. However, the most notable risk reduction was observed among participants who reported use of both aspirin and ibuprofen (HR=0.23,95\% CI: 0.06-0.92). The estimates were not significantly modified by adjustment for current smoking, gender, cardiovascular disease, time in study, or other factors. Excluding individuals who succumbed to the disease within 1 year of enrollment did not significantly alter these estimates.

\section{Discussion}

The results of the present study are similar to those of several other studies concluding that regular use of non-selective 
Table II. Hazard ratios for NSAIDs and fatal lung cancer among ever cigarette smokers enrolled in NHANES III.

\begin{tabular}{lcccr}
\hline Factor & Cohort & Cases & Hazard ratio & 95\% CI \\
\hline Ibuprofen & 858 & 21 & 0.52 & $0.33-0.82$ \\
Aspirin & 2,153 & 113 & 1.26 & $0.98-1.62$ \\
Acetaminophen & 1,795 & 69 & 0.88 & $0.66-1.16$ \\
Reference & 1,820 & 83 & 1.00 & 0.08 \\
\hline
\end{tabular}

Estimates were adjusted for other drugs, years in study, gender, current smoking and comorbid conditions (myocardial infarction, stroke, diabetes, or malignancies other than lung cancer). Exposure cohorts contain individuals reporting regular use of individual compounds at baseline. The reference cohort contains individuals reporting no use of any compound. NSAIDs, non-steroidal anti-inflammatory drugs; NHANES III, Third National Health and Nutrition Examination Study; CI, confidence interval.

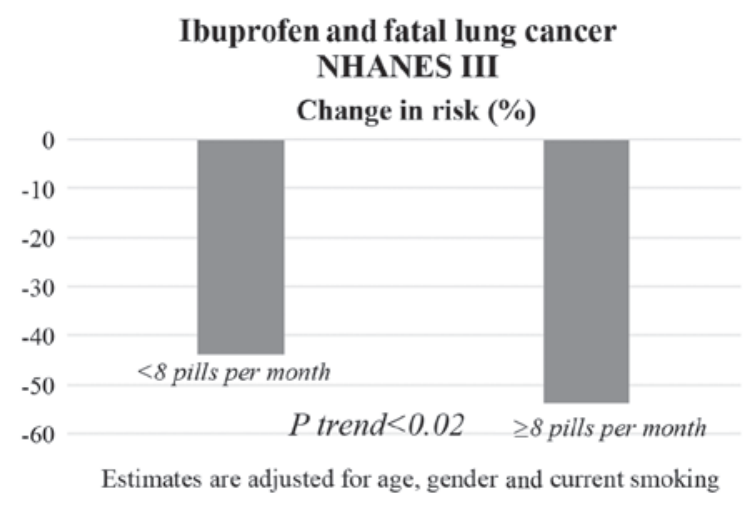

Figure 1. Lung cancer mortality risk among individuals reporting ibuprofen use compared with those reporting no use of non-steroidal anti-inflammatory drugs (NHANES III). NHANES III, Third National Health and Nutrition Examination Study.

NSAID agents was associated with a reduced risk of lung cancer $(3,4)$.

A major strength of this study is that NHANES III data were carefully collected and comprised a large cross-sectional sample that strongly represented the US population. The participants were not aware of the current study hypothesis when interviewed, and, therefore, potential response bias for the pain medication questions may have been avoided. Linkage with the National Death Index provided an objective measure of outcome.

Interpreting NHANES data was challenged by incomplete characterization of exposure (e.g., dose, duration and frequency of use) to more fully investigate associations with disease. For example, data on regular (325 mg) vs. low-dose (81 mg) aspirin use were not collected from NHANES III participants. Furthermore, the NHANES III only queried the participants regarding selected analgesic agents, and commonly used brands of aspirin (e.g., Alka Seltzer) and ibuprofen (e.g., Motrin) were not included in the list. These data are also subject to recall bias due to self-report.

The anti-inflammatory effects of ibuprofen, aspirin and other NSAIDs are primarily linked through the inhibition of cyclooxygenase (COX)-catalyzed prostaglandin biosynthesis (8). Two main genes that serve to regulate $\mathrm{COX}$ are a constitutive gene (COX-1) and its inducible isoform (COX-2) (9).

Molecular studies have demonstrated that COX-2 is overexpressed in most types of human cancer, including lung cancer, and that COX-2 inhibition reduces tumor growth and development. The blockade of COX-2 interferes with carcinogenesis by reducing mutagenesis, angiogenesis and cell division, as well as by stimulating apoptosis of malignant cells (10-12).

Our findings add to the evidence indicating that COX-2 expression may be a significant factor in lung carcinogenesis and, reciprocally, that NSAIDs that inhibit COX-2 may have chemopreventive benefits. Further studies are required to elucidate the dosage, duration, side effects and cost-effectiveness of such agents in the chemoprevention of lung cancer (13-15).

In conclusion, prospective data from NHANES III were analyzed to estimate the associations of aspirin, ibuprofen and acetaminophen with fatal lung cancer. Among adults with a history of past or current smoking, ibuprofen intake was associated with a $48 \%$ reduced risk of death from lung cancer, whereas the effects of aspirin and acetaminophen were not statistically significant.

\section{Acknowledgements}

The present study was partially supported by grant P30 CA16058 from the National Cancer Institute. Dr Carbone reports personal fees/honoraria received from AstraZeneca, Bayer Health Care, Biocept, Biothera, Boehringer, Ingelheim, Bristol Myers-Squibb, Clovis Oncology, Merck, Novartis, Peregrine Pharmaceuticals, Pfizer, Synta Pharmaceuticals and Teva Pharmaceuticals.

\section{References}

1. Ferlay J, Soerjomataram I, Ervik M, Dikshit R, Eser S, Mathers C, Rebelo M, Parkin DM, Forman D and Bray F: GLOBOCAN 2012 v1.0. Cancer Incidence and Mortality Worldwide. IARC Cancer Base No. 11. International Agency for Research on Cancer. Lyon, France, 2013.

2. Harris RE: Epidemiology of Lung Cancer. In: RE Harris. Global Epidemiology of Cancer. Jones \& Bartlett Learning, Burlington, MA, pp39-58, 2016.

3. Harris RE: Ibuprofen in the prevention and therapy of cancer. In: Ibuprofen, Discovery, Development and Therapeutics. Rainsford KD (eds). John Wiley \& Sons, West Sussex, pp518-546, 2015.

4. Khuder SA, Herial NA, Mutgi AB and Federman DJ: Nonsteroidal anti-inflammatory drug use and lung cancer: A meta-analysis. Chest 127: 748-754, 2005.

5. National Center for Health Statistics. Plan and operation of the Third National Health and Nutrition Examination Survey, 1988-94. Vital and Health Statistics I 32: 1-416, 1994. 
6. U.S. Department of Health and Human Services. Third National Health and Nutrition Examination Survey, NHANES III Examination Data File. Public Use Data File Documentation Number 76200. Hyattsville, MD, Centers for Disease Control and Prevention, 1996.

7. National Center for Health Statistics. Office of Analysis and Epidemiology, The Third National Health and Nutrition Examination Survey (NHANES III) Linked Mortality File, Mortality follow-up through 2006: Matching Methodology. Hyattsville, Maryland, 2009.

8. Rainsford KD: Pharmacology and toxicology of ibuprofen. In: Ibuprofen, Discovery, Development and Therapeutics. Rainsford KD (eds). John Wiley \& Sons, West Sussex, pp132-236, 2015.

9. Herschman HR: Regulation of prostaglandin synthase-1 and prostaglandin synthase-2. Cancer and Metas Rev 13: 241-256, 1994.

10. Balkwill $\mathrm{F}$ and Mantovani A: Inflammation and cancer: Back to Virchow? Lancet 357: 539-545, 2001.
11. Coussens LM and Werb Z: Inflammation and cancer. Nature 420: 860-867, 2000

12. Harris RE: Cyclooxygenase-2 (cox-2) and the inflammogenesis of cancer. Subcell Biochem 42: 93-126, 2007.

13. Shiff SJ and Rigas B: The role of cyclooxygenase inhibition in the antineoplastic effects of nonsteroidal anti-inflammatory drugs (NSAIDs). J Exp Med 190: 445-450, 1999.

14. Harris RE, Beebe-Donk J and Alshafie GA: Cancer chemoprevention by cyclooxygenase 2 (COX-2) blockade: Results of case control studies. Subcell Biochem 42: 193-212, 2007.

15. Olsen JH, Friis S, Poulsen AH, Fryzek J, Harving H, Tjønneland A, Sørensen HT and Blot W: Use of NSAIDS, smoking and lung cancer risk. Br J Cancer 98: 232-237, 2008. 